\title{
Inherent Structures, Configurational Entropy and Slow Glassy Dynamics
}

\author{
A. Crisanti $\dagger$ and F. Ritort $\ddagger$ \\ † Dipartimento di Fisica, Università di Roma "La Sapienza" and \\ Istituto Nazionale Fisica della Materia, Unità di Roma \\ P.le Aldo Moro 2, I-00185 Roma, Italy \\ † Physics Department, Faculty of Physics \\ University of Barcelona, Diagonal 647, 08028 Barcelona, Spain
}

\begin{abstract}
We give a short introduction to the inherent structure approach, with particular emphasis on the Stillinger and Weber decomposition, of glassy systems. We present some of the results obtained in the framework of spin-glass models and Lennard-Jones glasses. We discuss how to generalize the standard Stillinger and Weber approach by including the entropy of inherent structures. Finally we discuss why this approach is probably insufficient to describe the behavior of some kinetically constrained models.
\end{abstract}

PACS numbers: 64.70.Pf, 75.10.Nr, 61.20.Gy, 82.20.Wt 


\section{Introduction}

If we are asked what is a glass most probably we will think about a window-glass. The glassy state is, however, rather common in nature and many, apparently unrelated, systems such as structural glasses, spin glasses, disordered and granular materials or proteins among others, presents what is called a glassy behaviour. All these systems have as common feature a dramatic slowing down of the equilibration processes when some control parameter, e.g., the temperature or density, is varied. The equilibration process is frequently non-exponential, and correlation functions show power-law and stretched exponential behavior as opposed to a simple exponential decay. As the characteristic relaxation time may change of several orders of magnitude, it easily exceeds the observation time. The residual very slow motion leads to a non-equilibrium phenomena which changes the properties of the systems, a process commonly called aging. The greatest difficulty in understanding the slow relaxation glassy dynamics is that a general non-equilibrium theory to deal with this class of systems is still missing and approximations to this problem remain partial. They usually work either in a limited range of time scales or in a limited range of temperatures (for instance, mode-coupling theory 远).

Following the ideas formulated more than 30 years ago by Goldstein [2], a convenient framework for understanding the complex phenomenology of glassy systems is provided by the energy landscape analysis. The trajectory of the representative point in the configuration space can be viewed as a path in a multidimensional potential energy surface. The dynamics is therefore strongly influenced by the topography of the potential energy landscape: local minima, barriers, basins of attraction an other topological properties all influence the dynamics.

The idea of Goldstein, formulated at a qualitative level, was formalized years later

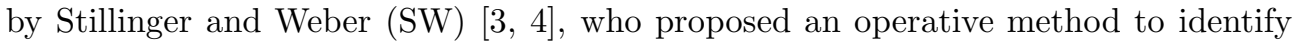
basins of the potential energy surface of super-cooled liquids. The recipe is rather simple: the set of all configurations connected to the same local energy minimum by a steepest-descent path uniquely defines the basin associated with this minimum, which Stillinger and Weber named Inherent Structure (IS) to stress its intrinsic nature.

The physical motivation behind their proposal follows from the observation that the potential energy surface of a super-cooled liquid contains a large number of local minima. Therefore the time evolution of the system can be seen as the result of two different processes: thermal relaxation into basins (intra-basin motion) and thermally activated potential energy barrier crossing between different basins (interbasin motion). When the temperature is lowered down to the order of the critical Mode Coupling Theory (MCT) temperature $T_{M C T}$ the typical barrier height is of the order of the thermal energy $k_{B} T_{M C T}$, and the inter-basin motion slows dominating the relaxation dynamics. If the temperature is further reduced the relaxation time eventually becomes of the same order of the physical observation time and the system falls out of equilibrium since there is not enough time to cross barriers and equilibrate. This define the experimental glass transition temperature $T_{g}$.

With this picture in mind it is natural to view the IS as the natural elements to describe the slow glassy dynamics. If we think of the glassy systems as a dynamical system, then the SW decomposition is a mapping of the true dynamics at a given temperature onto the IS dynamics. This approach is rather appealing since leads naturally to universality: all glassy systems with similar IS dynamics must have similar glassy behaviour. 
The increase of computational power has significantly improved the analysis of the energy surface and IS analysis of the energy surface has been done for several systems. The results are both positive and negative, indeed while the IS formalism has been successful for the description of the off-equilibrium dynamics and the FDT violations in structural glass models 5, 6, 2, 8, 9, it fails for some kinetically constrained glassy systems 10 .

To understand this success/failure we have to analyse the idea behind the SW approach. The main question we would like to answer is: what is a good description for the long time slow glassy dynamics? The natural approach is to look for some "reduced dynamics" which includes only those details of the full dynamics relevant on the long time scales. This obviously implies a coarse graining of the phase space. For example within the Mori's projectors method used to derive the MCT, the phase space is coarse grained by projecting it onto the the subspace spanned by the "slow variables". It is clear that even if the phase space can be always coarse grained, not all possible coarse graining will lead to a relevant reduced dynamics. This is a well known problem in the theory of dynamical systems, where the associated reduced dynamics is called symbolic dynamics. Indeed from the theory of dynamical systems we know that a symbolic dynamics gives a good description of the full dynamics only if the mapping between the full phase space and the coarse grained one defines what is called a generating partition, see e.g. Ref. [11]. In general for a generic systems not only it is not trivial to demonstrate that a generating partition exists but, even when it does exist, its practical identification remains a highly non-trivial task so that we can answer to this question only at posteriori. We first define a partition and then check if this reproduces the desired features of the dynamics.

The SW mapping identifies configuration in a IS-basin with the IS itself. Therefore to be a plausible mapping the systems must spend a lot of time inside the basin. Under this assumption the dynamics on time scales larger than the typical residence time inside a IS-basin could be quite well described by the IS dynamics. This scenario is typical, e.g., of a many valley energy landscape with activated dynamics. This, however, is only one of the plausibility conditions since other requirements on the dynamics must be satisfied, as discussed later in this work.

To illustrate our discussion we shall report results from numerical simulations of finite-size fully-connected Ising-spin Random Orthogonal Model (ROM) [12] and binary mixture of Lennard-Jones (BMLJ) [13]. The first one, belonging to the $p$-spin class [14], is a fully connect Ising spin glass model with a random orthogonal interaction matrix whose high-temperature dynamics is described in the thermodynamic limit by the MCT 15. The second one, a typical system used for studying the structural glass transition, is a system composed by a mixture of type $A$ and type $B$ particles interacting via a Lennard-Jones potential. One of the main advantages is that with a suitable choice of the Lennard-Jones potential parameters for $A A, A B$ and $B B$ interactions the system does not crystallize simplifying the analysis of the glass transition.

\section{The Stillinger and Weber decomposition}

\subsection{The $S W$ configurational Entropy}

The recipe of the Stillinger and Weber decomposition is rather simple [3]: the set of all configurations connected to the same local energy minimum (IS) by a steepest-descent 
path uniquely defines the basin associated with the minimum. The phase space is then partitioned into a disjoint set of basins, usually labeled by $e_{\mathrm{IS}}$ the energy of the IS. Under broad assumptions, e.g., that boundaries between basins are sub-extensive, this decomposition covers almost all the energy surface and, collecting all IS with the same energy, the partition sum is written as sum of basin partition functions:

$$
Z_{N}(T) \simeq \int d e \exp N\left[s_{c}(e)-\beta f_{b}(T, e)\right]
$$

where $N s_{c}(e)$ account for the entropic contribution arising from the number of basins with energy $e_{\mathrm{IS}}=e$. We shall call $s_{c}(e)$ the $S W$ configurational entropy or complexity to distinguish it from other possible definitions taken from mean-field concepts [16, 17]. The term $f_{b}(T, e)$ is the typical free energy of the $e_{\mathrm{IS}}$ basins with energy $e_{\mathrm{IS}}=e$. If all $e_{\mathrm{IS}}=e$ basins have similar statistical properties then $f_{b}(T, e)$ is the free energy of the system when constrained in one characteristic $e_{\mathrm{IS}}$ basin with $e_{I S}=e$.

In equilibrium at each temperature $T=1 / \beta$ the system will visit $e_{\text {IS }}$ basin with probability, see eq. (位),

$$
P_{N}\left(e_{\mathrm{IS}}, T\right)=\exp N\left[s_{c}\left(e_{\mathrm{IS}}\right)-\beta f_{b}\left(T, e_{\mathrm{IS}}\right)\right] / Z_{N}(T)
$$

therefore, in the thermodynamic limit, it will populates mainly $e_{\mathrm{IS}}$ with energy $e(T)$ fixed by the condition

$$
s_{c}(e)-\beta f_{b}(T, e)=\text { maximum. }
$$

and the free energy (density) of the system becomes

$$
f(T)=f_{b}(T, e(T))-T s_{c}(e(T)) .
$$

The separation of the free energy into two contributions reflects the time-scale separation between inter and intra basin motions. Condition (3) is equivalent to that of $f(T)$ being minimal, i.e.,

$$
\frac{\partial f}{\partial e}=\frac{\partial f_{b}(T, e)}{\partial e}-T \frac{\partial s_{c}(e)}{\partial e}=0 .
$$

Note that the minimum condition follows from the balance between the contribution from the change with the energy of the shape of the basins $\left(\partial f_{b}(T, e) / \partial e\right)$ and its corresponding number $\left(\partial s_{c}(e) / \partial e\right)$.

Often it is useful to write the basin free energy as $f_{b}\left(T, e_{\mathrm{IS}}\right)=e_{\mathrm{IS}}+f_{v}\left(T, e_{\mathrm{IS}}\right)$ to evidenciate the contribution from the motion inside the $e_{\mathrm{IS}}$ basins. Indeed from (4) the average internal energy density reads $u(T)=\partial\left(\beta f_{b}\right) / \partial \beta=e(T)+\partial\left(\beta f_{v}\right) / \partial \beta$ where the first term is the (average) energy of $e_{\text {IS }}$ relevant at temperature $T$, while the second term is the contribution from fluctuations inside the $e_{\mathrm{IS}}$ basin. The contribution $f_{v}$ is called "vibrational".

The main advantage of the SW decomposition is that it can be easily transformed into a numerical algorithm, and the increase of the computational power has greatly pushed the IS analysis of the energy surface. The scheme, summarized in Figure 1 , follows directly from the definition.

First we equilibrate the system at a given temperature $T$, then starting from an equilibrium configuration the system is instantaneously quenched down to $T=0$ by decreasing the energy along the steepest descent path. The procedure is repeated several times starting from uncorrelated equilibrium configurations. In this way the IS are identified and quantities such as the $e_{\mathrm{IS}}$ probability distribution or $e(T)$ computed. 


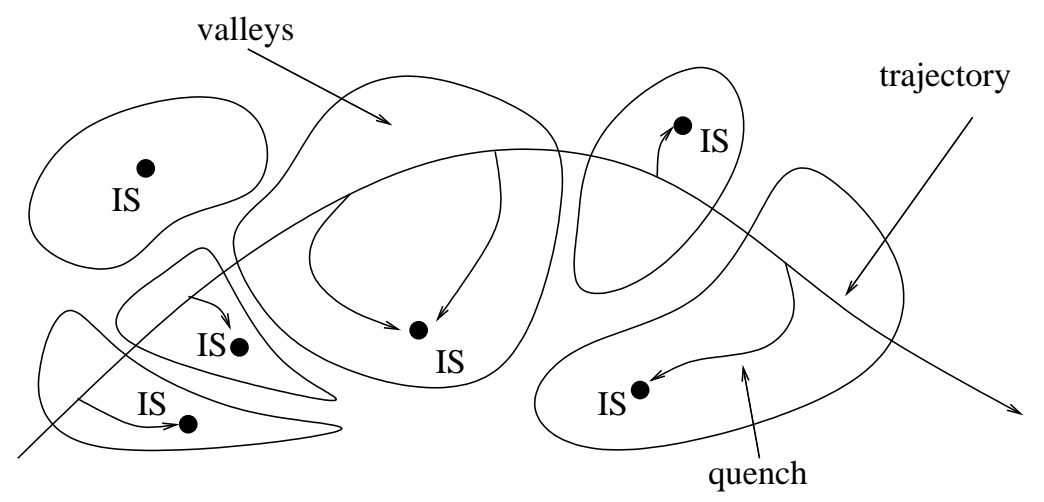

Figure 1. Stillinger and Weber decomposition

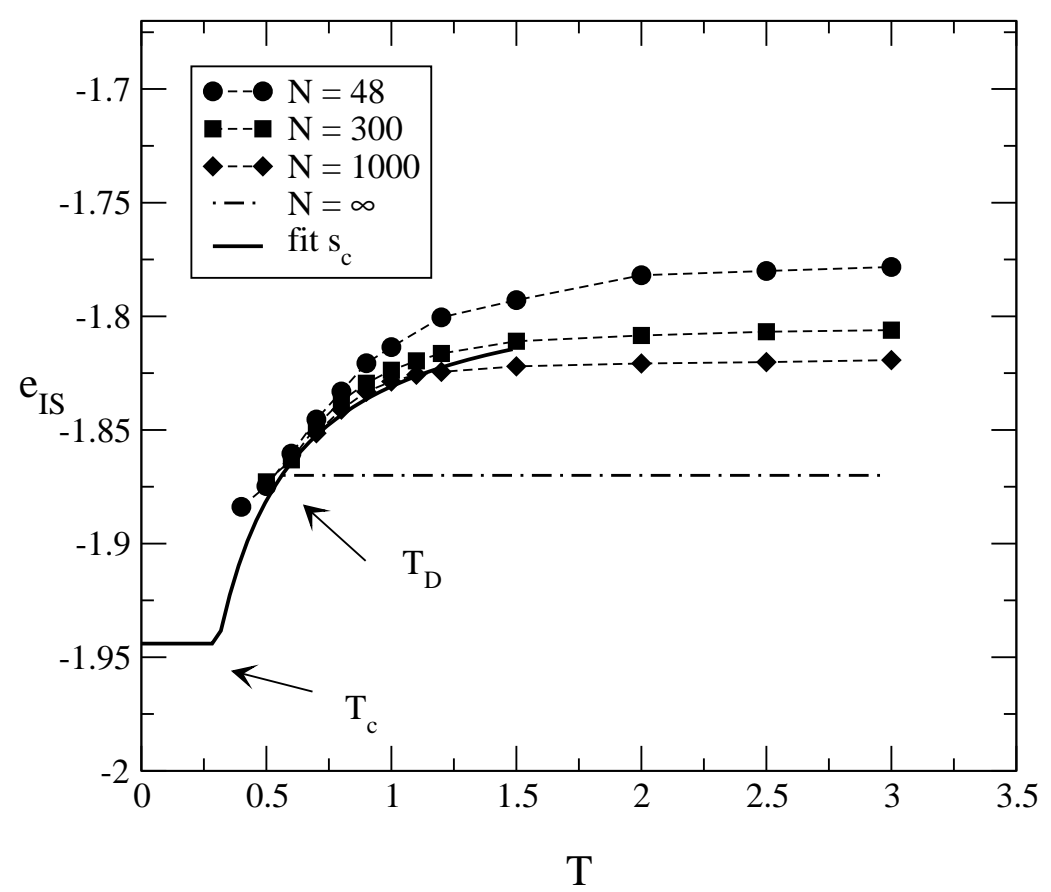

Figure 2. Temperature dependence of $e(T)$ for the Random Orthogonal Model with $N=48$ (square), $N=300$ (circle) and $N=1000$ (diamond). The horizontal line is the $N \rightarrow \infty$ limit. The arrows indicate the critical dynamic temperatures $T_{D}$ (dynamic or Mode Coupling) and $T_{c}$ (static or Kauzman). The line is the curve obtained from the configurational entropy for large $N$. [see also Ref. [6]].

In Figure 2 we report $e(T)$ as a function of temperature $T$ for the Random Orthogonal Model (ROM) of different sizes, while in Figure 3 the same quantity is shown for a binary mixture of Lennard-Jones (BMLJ) particles.

From the figures we see a sharp drop in the $e_{\mathrm{IS}}$ energy as temperature is lowered. For all systems, both with discrete or continuous variables, displaying a fragile glass transition studied so far the drop turns out to be strongly correlated with the onset of slow dynamics 18]. Indeed the decreasing of the $e_{\text {IS }}$ value is a clear indication that 
the system explores deeper and deeper basins.

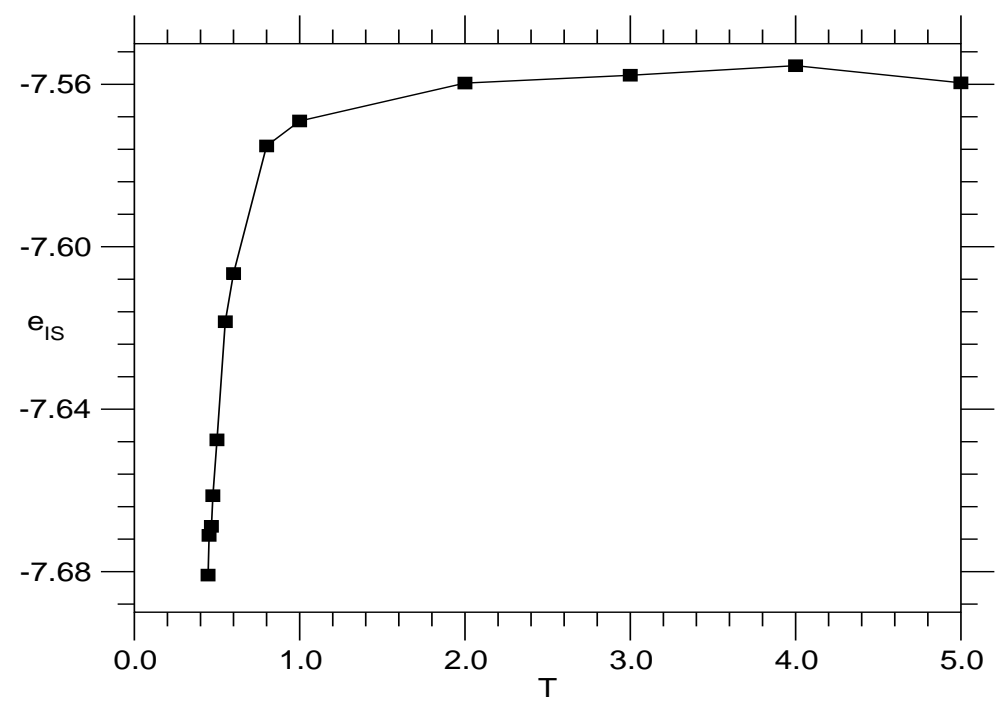

Figure 3. Temperature dependence of $e(T)$ for the a binary (80:20) mixture of Lennard-Jones particles. Simulations were done for 1000 particles at a fixed density of 1.2 [Data courtesy of W. Kob, F. Sciortino and P. Tartaglia; see also Ref. [19]].

From the knowledge of $e_{\mathrm{IS}}$ distribution we can reconstruct the SW complexity $s_{c}(e)$ simply inverting eq. (2):

$$
s_{c}(e)=\ln P_{N}(e, T)+\beta e+\beta f_{v}(T, e)+\ln Z_{N}(T)
$$

If energy dependence of $f_{v}(T, e)$ can be neglected, then it is possible to superimpose the curves for different temperatures. The resulting curve is, except for an unknown constant, the SW complexity $s_{c}(e)$. This is shown for the ROM in Figure 1 panel (a). The data collapse is rather good for $e<-1.8$, indicating that above the energy dependence of $f_{v}(T, e)$ cannot be neglected. The line is the quadratic best fit which gives the value $e_{c} \simeq-1.944$ for the critical energy where $s_{c}(e)$ vanishes in good agreement with the theoretical result $e_{1 r s b}=-1.936$ [12].

Direct consequence of $f_{v}(T, e) \simeq f_{v}(T)$ for $e<-1.8$ is that it drops out from eq. (5) so that the minimum condition simply reads:

$$
\frac{d s_{c}(e)}{d e}=\frac{1}{T}
$$

From this relation, by integrating the $T$ dependence of $d e / T$, we can compute $s_{c}$ as a function of $T$. The result obtained using the data of Figure 2 is shown in Figure 1 panel (b). The line is the result valid for large $N$ obtained using the quadratic best fit of panel (a).

As discussed above the vibrational contribution $f_{v}$ follows from the motion inside the $e_{\mathrm{IS}}$ basins. Its independence from $e_{\mathrm{IS}}$ means that all basins are equivalent, i.e., have the same shape. In general this is not the case and its contribution must be included. For systems with continuous variables, as for example BMLJ particles, at 


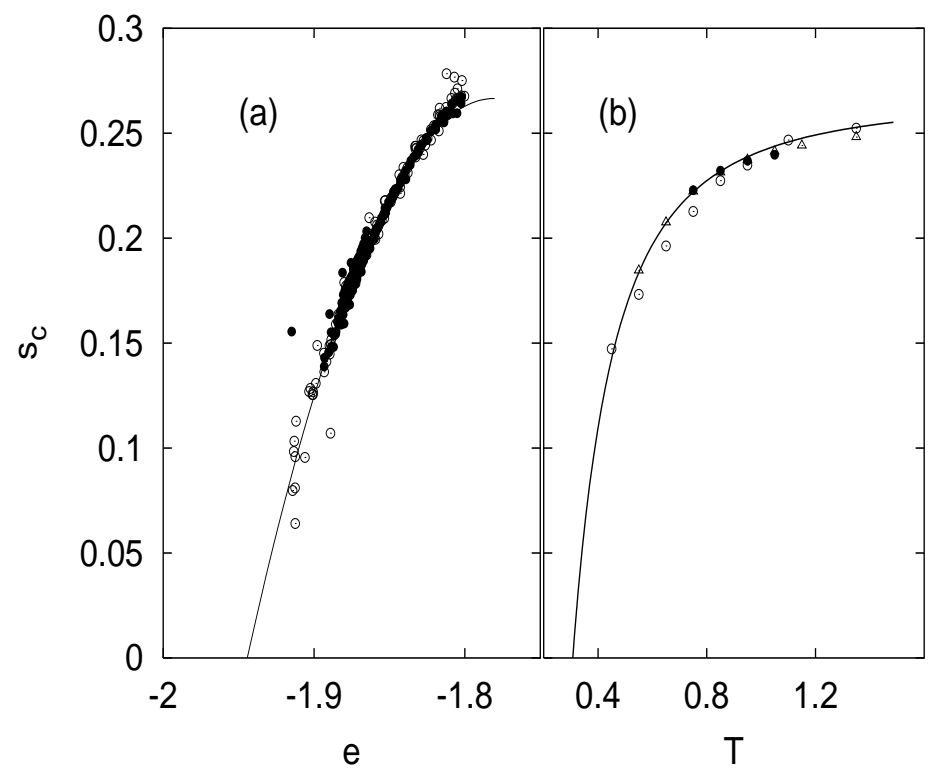

Figure 4. (a) Configurational entropy as a function of energy for the ROM. The data are from system sizes $N=48$ (empty circle) and $N=300$ (filled circle), and temperatures $T=0.4,0.5,0.6,0.7,0.8,0.9$ and 1.0. For each curve the unknown constant has been fixed to maximize the overlap between the data and the theoretical result 12. The line is the quadratic best-fit. (b) Configurational entropy density as a function of temperature. The line is the result from the bestfit of $s_{c}(e)$ while the symbols are the results from the temperature integration of eq. (1) for $N=48$ (empty circle), $N=300$ (empty triangle) and $N=1000$ (filled circle). [see also Ref. 6]].

low $T f_{v}$ can be calculated in the harmonic approximation by expanding the energy about the IS configuration:

$$
f_{v}\left(T, e_{I S}\right)=k_{B} T \sum_{i=1}^{3 N-3} \ln \left[\hbar \omega_{i}\left(e_{\mathrm{IS}}\right) / k_{B} T\right]
$$

where $\omega_{i}\left(e_{\mathrm{IS}}\right)$ is the frequency of the $i$-th normal mode in the $e_{\mathrm{IS}}$ basin which in general depends on the specific IS configuration, i.e., different IS with the same $e_{\mathrm{IS}}$ may have different normal modes. If all basins had the same curvature, then $f_{v}$ would be only function of $T$ and we are back to the previous case. In the BMLJ system, basins with different $e_{\mathrm{IS}}$ have different curvatures [9] and hence, at difference with the ROM, $f_{v}$ is a function of both $T$ and $e_{\mathrm{IS}}$.

\subsection{Violation of FDT and Effective Temperature}

More informations on the structure of the energy surface can be obtained from non-equilibrium relaxation processes. We shall consider here the non-equilibrium behaviour of the system following an instantaneous quench from an equilibrium state a temperature $T_{i}$ above the glass transition $T_{g}$ to a temperature $T_{f}$ below it.

For temperature close to the mode coupling critical temperature, where the intrabasin and inter-basin time scales become well separated, the vibrational intra-basin 
dynamics quickly thermalize to the thermal bath temperature $T_{f}$. The thermalization of the entire system is instead rather slow, being dominated by the inter-valley processes. Therefore the fast equilibration of the intra-basin degrees of freedom is followed by a much slower process during which the system populates deeper and deeper $e_{I S}$ levels.

In the right panel of Figure 5 we show the average $e_{\text {IS }}$ energy as a function of time after the quench for the ROM. The figure reveals that the relaxation process can be divided into two different regimes. A first regime with a power law independent of $T_{f}$, and a second regime with a power law independent of both $T_{i}$ and $T_{f}$. The final temperature $T_{f}$ controls the cross-over between the two regimes. A similar behavior has been observed in molecular dynamics simulations of super-cooled liquids [19].

The two regimes are associated with different relaxation processes. In the first part the system has enough energy and relaxation is mainly due to path search out of basins through saddles of energy lower than $k_{B} T_{f}$. This part depends only on the initial equilibrium temperature $T_{i}$ and should slow down as $T_{i}$ decreases since, as reasonable, lower states are surrounded by higher barriers. This process stops when all barrier heights become of $O\left(k_{B} T_{f}\right)$ and relaxation slows down since it must proceed only via activated inter-valley processes.

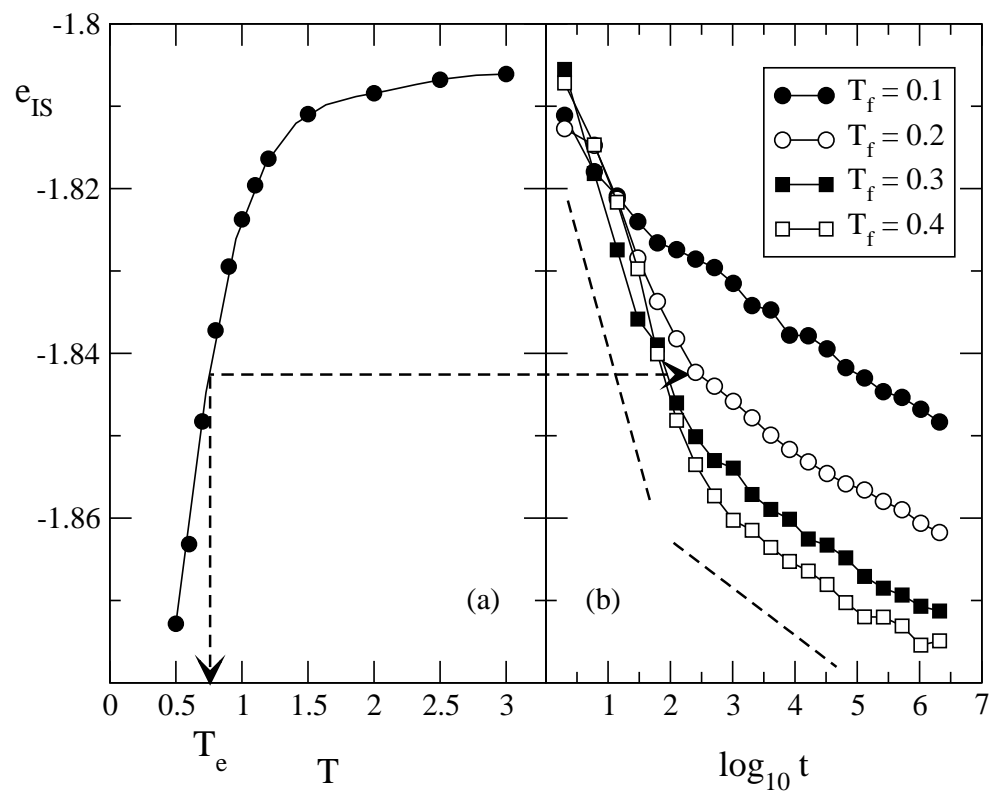

Figure 5. Panel (b): Average inherent structure energy for the ROM as function of time for initial equilibrium temperatures $T_{i}=3.0$ and final temperatures $T_{f}=0.1,0.2,0.3$ and 0.4 . The average is over 300 initial configurations. The system size is $N=300$. The lines denotes the two regimes. Panel (a): Equilibrium average $e_{\text {IS }}$ a function of temperature. The arrows indicate the construction of the effective temperature $T_{e}\left(e_{\mathrm{IS}}\right)$. [see also Ref. [7]].

Under the assumption of a fast equilibration of the intra-basin motion, we can define an effective temperature $T_{e}$ as the temperature the system would have when populating the basins of depth $e_{\mathrm{IS}}$. The temperature $T_{e}$ can be obtained from (5) with the vibrational contribution evaluated at the bath temperature $T_{f}$, since we 
assume local equilibrium of the intra-basin motion, and resolving for $T$ [9]:

$$
T_{e}\left(e_{\mathrm{IS}}, T_{f}\right)=\frac{1+\left(\partial / \partial e_{\mathrm{IS}}\right) f_{v}\left(T_{f}, e_{\mathrm{IS}}\right)}{\left(\partial / \partial e_{\mathrm{IS}}\right) s_{c}\left(e_{\mathrm{IS}}\right)}
$$

The effective temperature $T_{e}$ for the BMLJ is shown in in Figure 6. If $f_{v}$ is not a

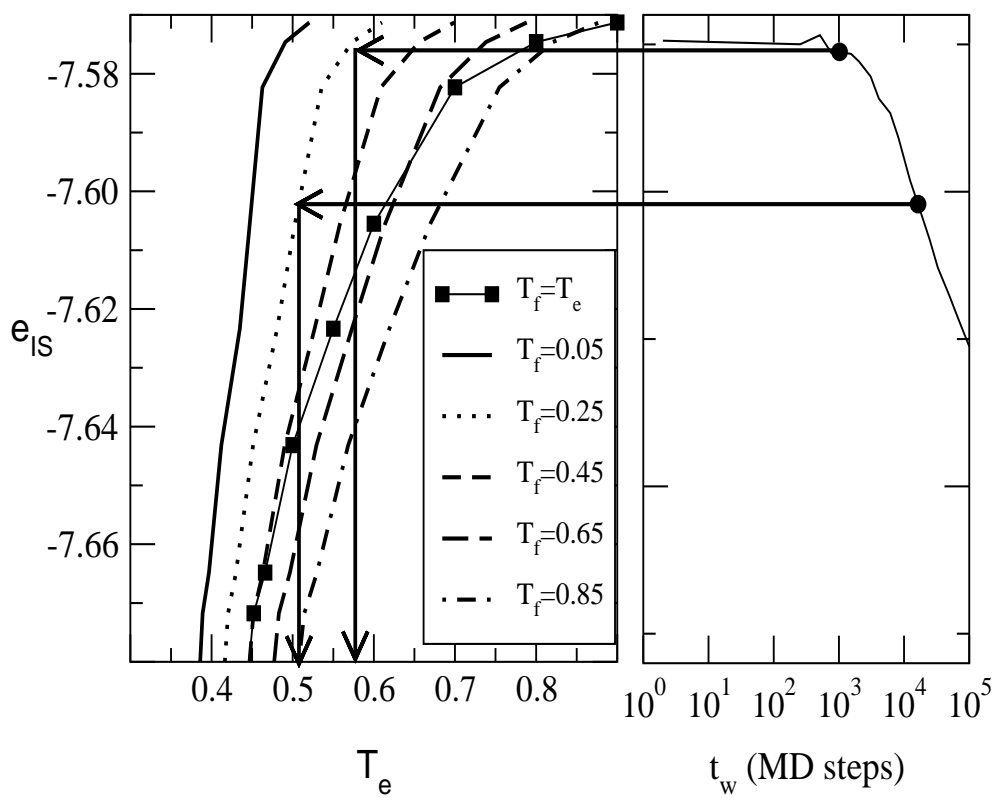

Figure 6. Left: Solutions of Eq. (9) for several values of $T_{f}$ for the BMLJ system. Right: $e_{\text {IS }}$ as a function of time, following the temperature quench. The arrows show graphically the procedure which connects the $e_{\mathrm{IS}}(t)$ value to $T_{e}$ value, gnce $T_{f}$ is known. [Data courtesy of F. Sciortino and P. Tartaglia, see also Ref. [6].

function of $e_{\mathrm{IS}}$, as in the case of ROM, the above equation reduces to [6, 8, 8]

$$
T_{e}\left(e_{\mathrm{IS}}\right)^{-1}=\frac{\partial s_{c}\left(e_{\mathrm{IS}}\right)}{\partial e_{\mathrm{IS}}}
$$

and curves with different $T_{f}$ coincide, see Figure 5 . In the non-equilibrium relaxation process the value of $e_{\mathrm{IS}}$ will vary with time making $T_{e}$ a function of time, since the r.h.s. of eqs. (9) and (10) must be evaluated at $e_{\mathrm{IS}}(t)$. For each time $t$ the value of $T_{e}(t)$ can then be obtained graphically as shown in Figures 5 and 6 .

The predictions of the quasi-equilibrium assumption can be tested by studying the response of the system after a quench to $T_{f}$ at time $t=0$ to a perturbation switched on at some later time $t_{w}$. In the linear response regime the average value of any observable $A$ at time $t$ after a perturbation field $h_{A}$ conjugated to it is applied to the system at $t_{w}<t$ is $\langle A(t)\rangle=\chi_{\mathrm{ZFC}}\left(t, t_{w}\right) h_{A}$, where the Zero Field Cooled susceptibility $\chi_{\mathrm{ZFC}}\left(t, t_{w}\right)$ is related to the $A A$ correlation function through the famous FluctuationDissipation formula 20]

$$
\left.\chi_{\mathrm{ZFC}}\left(t, t_{w}\right)=\frac{1}{T}\left[\langle A(t) A(t)\rangle-\left\langle A(t) A\left(t_{w}\right)\right]\right\rangle\right]\left.\right|_{h_{A}=0} .
$$

which predicts that the response be proportional to $T^{-1}$. Under the assumption that the intra-valley motion quickly thermalizes to the thermal bath temperature $T_{f}$, while 
the thermalization of the entire system is dominated by the slow inter-valley processes, it follows that for short times $T=T_{f}$ since the intra-basin motion is probed, while for long times $T=T_{e}$ since now is the inter-basin to be probed. The first regime corresponds to the region where the correlation function assumes values between the equal time and the plateau values, while the second where it assumes values below the plateau value.

In Figure 7 we show the response versus correlation plot for the ROM. The analogous plot for the BMLJ is in Figure 8. As follows from both figures for short times the plot is linear with the expected $T_{f}^{-1}$ slope, properly describing the equilibrium condition of the intra-basin dynamics with the external bath. At larger times, the inter-basin motion sets-in and the slope becomes $T_{e}^{-1}$, in very good agreement with the value predicted by eq. (9).

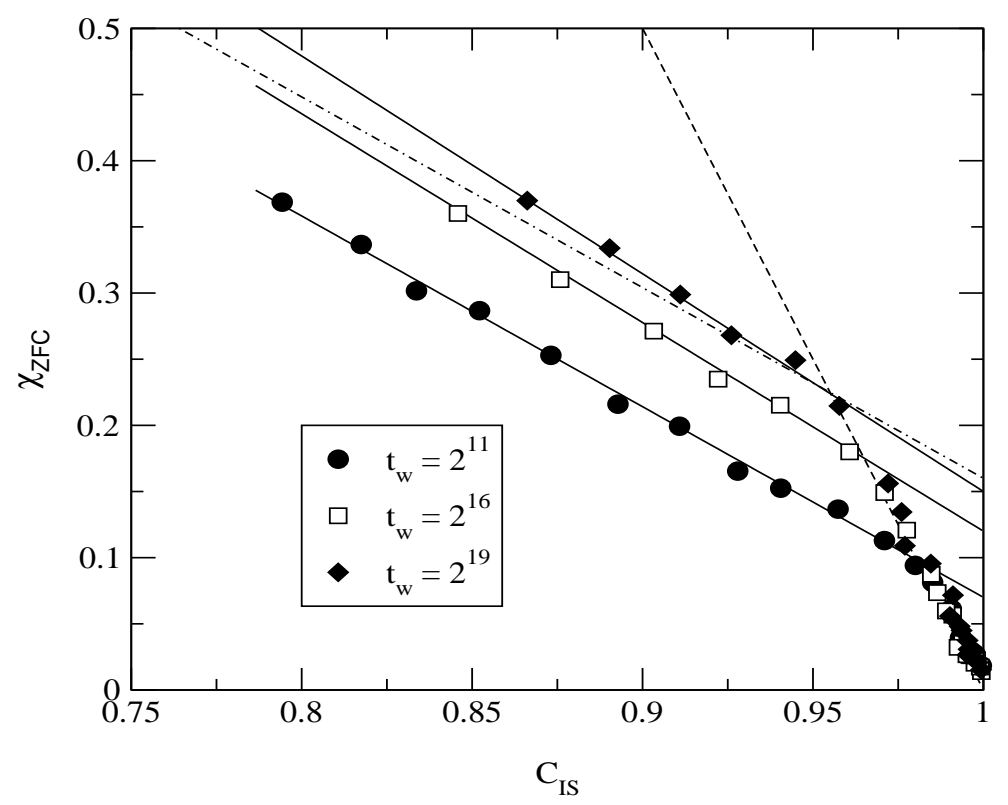

Figure 7. Integrated response function as a function of IS correlation function, i.e, the correlation between different IS configurations, for the ROM. The dash line has slope $T_{f}^{-1}=5.0$, while the full lines are the prediction 10$): T_{e}\left(2^{11}\right) \simeq 0.694$, $T_{e}\left(2^{16}\right) \simeq 0.634$ and $T_{e}\left(2^{19}\right) \simeq 0.608$. The dot-dashed line is $T_{e}$ for $t_{\mathrm{w}}=2^{11}$ drawn for comparison. [see also Ref. 7]].

\section{Beyond the Stillinger and Weber projection: the free energy landscape}

In the previous sections the main effort has been to characterize glassy dynamics by studying the structure of minima of the potential energy landscape. Nevertheless, as has been already said valleys are not only characterized by the energy at their bottom but also by the size of the basins of attraction. The decomposition proposed by Stillinger and Weber is meaningful if the typical time to explore a given IS or valley only depends on the energy of that valley (defined, for instance by the energy $e_{\mathrm{IS}}$ of its associated minimum). But one can imagine a situation where IS with the same energy have completely different basins of attraction. In that case the probability to explore 


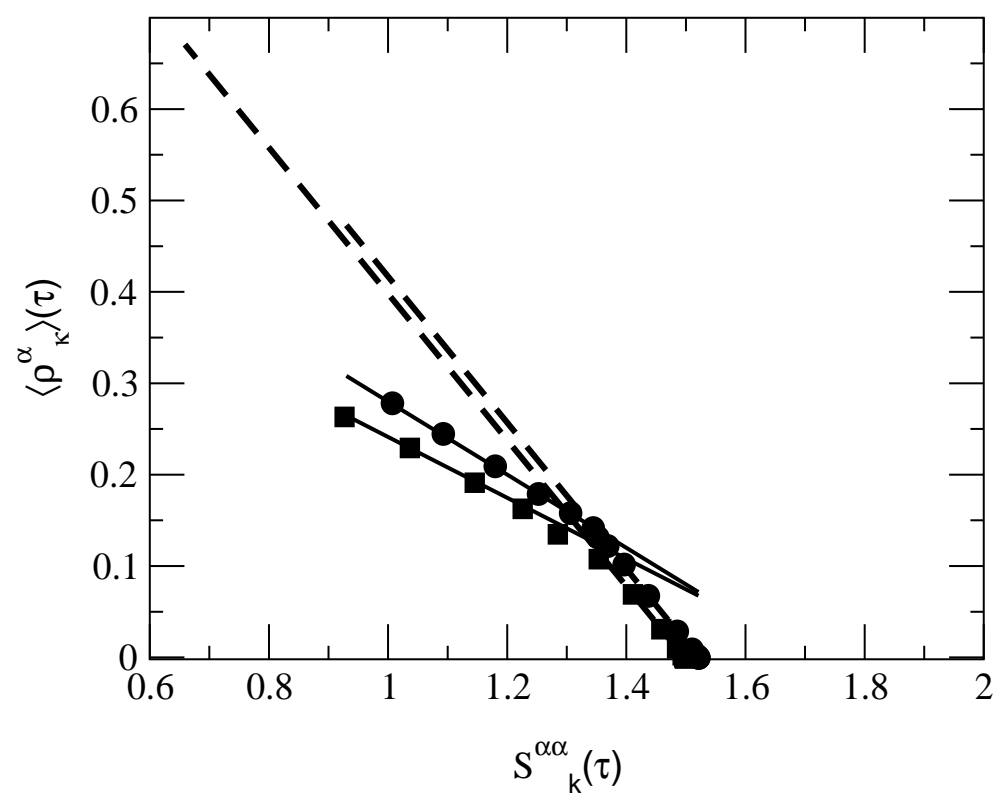

Figure 8. Response $\left\langle\rho_{\mathbf{k}}^{\alpha}(\tau)\right\rangle$ versus the dynamical structure factor $S_{\mathbf{k}}^{\alpha \alpha}(t) \equiv$ $\left\langle\rho_{\mathbf{k}}^{\alpha}(t) \rho_{\mathbf{k}}^{\alpha *}(0)\right\rangle$, where $\rho_{\mathbf{k}}^{\alpha}$ is the Fourier transform component of the density of $\alpha=A, B$ particles at wave-vector $\mathbf{k}$, for the Binary Mixture Lennard-Jones particles system for $T_{i}=0.8, T_{f}=0.25$ and two waiting times $t_{w}=1024$ (square) and $t_{w}=16384$ (circle). Dashed lines have slope $T_{f}^{-1}$ while thick lines have slope $T_{e}^{-1}$. [Data courtesy of F. Sciortino and P. Tartaglia, see also Ref. 9]].

a given IS not only depends on its energy but also on the size of the basin of attraction or its associated entropy. The most natural approach in which these considerations are properly taken into account is to assume that every IS is characterized by its free energy $F_{\mathrm{IS}}(T)$ defined as:

$$
\exp \left(-\beta F_{\mathrm{IS}}(T)\right)=\sum_{\mathcal{C} \in I S} \exp (-\beta E(\mathcal{C}))
$$

which corresponds to the free energy of a portion of the whole phase space containing all configurations belonging to the specific IS. We can now extend 21] the ideas of Stillinger and Weber to include the free energy in the formulation by using the following equiprobability assumption: when the system is in equilibrium at a given temperature, valleys with the same free energy have the same probability to be explored. Both the number of configurations contained in each valley and the number $g(F, T)$ of valleys with a given free energy grow exponentially with the size of the system leading to a proper thermodynamic formulation in the large volume limit. The equilibrium partition function can be written in terms of the IS free energies (12) as:

$$
\begin{aligned}
\mathcal{Z} & =\sum_{\mathcal{C}} \exp (-\beta E(\mathcal{C}))=\sum_{\mathrm{IS}} \sum_{\mathcal{C} \in \mathrm{IS}} \exp (-\beta E(\mathcal{C})) \\
& =\sum_{\mathrm{IS}} \exp \left(-\beta F_{\mathrm{IS}}(T)\right)
\end{aligned}
$$


At a given temperature, the average free energy among all valleys is determined by a balance between the probability to explore valleys with free $F$ (proportional to the Boltzmann factor $\exp (-\beta F))$ and the number $g(F, T)$ of valleys with that free energy. Hence, the equilibrium free energy is given by

$$
\begin{aligned}
\exp \left(-\beta F_{\text {eq }}\right) & =\sum_{\mathrm{IS}} \exp \left(-\beta F_{\mathrm{IS}}(T)\right)=\sum_{F} g(F, T) \exp (-\beta F) \\
& =\sum_{F} \exp \left(S_{c}(F, T)-\beta F\right)=\sum_{F} \exp (-\beta \Phi(F, T))
\end{aligned}
$$

where $S_{c}(F, T)=\log (g(F, T))$ defines the configurational entropy while the function $\Phi(F, T)=F-T S_{c}(F, T)$ is the thermodynamic potential associated to it. Because $\Phi$ is an extensive quantity, in the large-volume limit, the leading contribution to (14) is determined by the minimum of $\Phi(F, T)$ as function of $F$,

$$
\begin{aligned}
& F_{\mathrm{eq}}(T)=\Phi\left(F^{*}, T\right)=F^{*}-T S_{c}\left(F^{*}, T\right) \\
& \frac{1}{T}=\left.\frac{\partial S_{c}(F, T)}{\partial F}\right|_{F=F^{*}} .
\end{aligned}
$$

Note that the average free energy $F^{*}$ does not coincide with the equilibrium free energy $F_{\text {eq }}(T)$ but it is always higher, $F^{*}=F_{\text {eq }}(T)+T S_{c}\left(F^{*}, T\right)$, the difference being the configurational entropy evaluated at $F^{*}$.

It is interesting to note the parallelism between this coarse-grained description and the standard equilibrium theory. In that case, the relevant entities are the configurations and the equilibrium energy is given by a balance between the Boltzmann factor $\exp (-\beta E)$ and the degeneration $g(E)=\exp (S(E))$ where $S(E)$ is the entropy. The relation $1 / T=\frac{\partial S(E)}{\partial E}$ yields the thermodynamics. Nevertheless, an important difference between the standard equilibrium theory and the present free energy valley decomposition must be stressed. Both the equilibrium entropy $S(E)$ and the Stillinger and Weber configurational entropy $S_{c}(E)$ are only functions on the energy while the configurational entropy $S_{c}(F, T)$ in this new formalism depends on both the free energy and the temperature. Therefore the configurational entropy turns out to be a more complicated object when expressed in terms of the free energy than in terms of the energy of the valleys.

A natural question arises now: what are the assumptions behind the validity of this free energy decomposition? The main assumption in Boltzmann theory is the equiprobability assumption, i.e., all configurations with identical energy have in equilibrium the same probability. This introduces the equilibrium measure used in ensemble theory which is at the roots of Statistical Mechanics. For glassy systems a similar idea is behind the physical meaning of the present free energy decomposition. One assumes 21] the validity of an equiprobability hypothesis stating that valleys with identical free energy have the same probability to be explored. This gives a flat measure in equilibrium which, if extended to the non-equilibrium case, is the analogous to the Edwards measure proposed in the context granular media [22, 23, 24]. The main difference between the Edwards measure and this new free energy measure is that the former occurs in a non stationary sheared situation [25] or even in an stationary one (for instance, under tapping [26]) at zero temperature while the latter occurs in a non-stationary relaxational regime at finite temperature. 
In a recent work [21] the validity of this free energy measure has been explicitly tested by studying the configurational entropy of simple models. This has been done introducing a probabilistic argument to compute the free energy of the IS. In equilibrium, the probability to explore a given IS, is given by $p_{\mathrm{IS}}(T)=$ $\exp \left(-\beta\left(F_{\mathrm{IS}}(T)-F_{\mathrm{eq}}(T)\right)\right)$. Then one can run a simulation and, after equilibrating, collect the number of times $N_{\text {IS }}$ that a particular IS is found among a total number of quenches $N_{\text {run }}$. This yields $p_{\text {IS }}(T)=N_{\text {IS }} / N_{\text {run }}$ from which we have an estimate of the IS free energy: $F_{\mathrm{IS}}(T)=-T \log \left(p_{\mathrm{IS}}(T)\right)+F_{\text {eq }}(T)$. By using this method the configurational entropy $S_{c}(F, T)$ has been computed in Ref. [21] for the ROM and the Sherrington and Kirkpatrick model, a model with a completely different energy surface topology [27]. The average free energy of the IS, $F^{*}$ can be obtained from the minimum of the potential $\Phi(F, T)$. The difference between the minimum $F^{*}(T)$ and the equilibrium free energy $F_{\text {eq }}(T)$ yields the configurational entropy at the given temperature $S_{c}\left(F^{*}, T\right)$. Moreover from the shape of $\Phi(F, T)$ it is possible to infer both the type of transition of the model, one-step or infinite-replica symmetry breaking, as well as the critical o Kauzman temperature. We note that as it happens for the Stillinger and Weber results discussed in the previous section, the method works only for finite-sized systems where the number of different IS is not too large.

The generalization of this free-energy scenario to the dynamics has been proposed in [28] where it has been proposed that, the effective temperature in structural glasses is related to its fragility. Moreover, the effective temperature is also given by the slope of the configurational entropy evaluated at the threshold free energy. This off-equilibrium scenario complements the new measure discussed before and offers a scenario for the glass transition driven by entropic barriers.

\section{Models with kinetical constraints.}

Another class of interesting models are kinetically constrained models. These models are characterized by a extremely simple thermodynamic behavior without any type of phase transition but with a complicated slow dynamics due the existence of kinetical constraints. The constraints are such that detailed balance and ergodicity are preserved despite the infinite energy barriers they introduce. In some sense they are similar to hard spheres models where some configurations are excluded form the configurational space although with much simpler static properties. The simplest example of these family of models is the kinetically constrained Ising paramagnet where there is no interaction between the spins (hence there is no critical point even at $T=0$ ) but some transitions are excluded in the dynamics. The description of these models within an IS formalism has been studied in detail in [10. Here we only want to make some general considerations about the validity of the IS description of the dynamics, for a more detailed discussion we refer to Ref. [10].

In general, in most of the dynamically constrained models the energy function can take only a discrete set of values, therefore there is accidental degeneracy in the density of states. One of the main problems related to this fact and discussed in Ref. [10] is that it is difficult to properly define the IS decomposition itself. The steepest descent procedure to map a configuration onto a valley is not well defined because it is not univocally defined. From a physical point of view this does not seem to be a serious problem. Suppose we add to the original Hamiltonian a random perturbation term $P$ which lifts the degeneration. If the system is stochastically stable (the dynamical behavior in the limit $P \rightarrow 0$ coincides with the dynamical behavior of the unperturbed 
system) then one can work out with the perturbed system and make the perturbation vanish at a later stage. Because the dynamics of the vast majority of glassy systems is probably stochastically stable we believe that this is not serious problem of the approach.

But there is another problem which seriously compromises the validity of the IS approach. One can show that the IS decomposition is completely identical for some models with completely different dynamical constraints, so that the SW configurational entropy is exactly the same. Because the dynamics of these models is known to be extremely different it is clear that such configurational entropy cannot describe their relaxational dynamics. Obviously, to cope with this problem one can go further and describe the dynamical behavior in terms of the configurational entropy but now defined in terms of the free energy. This route could eventually solve the problem because now, although the energy of the different IS is identical for all models, the entropic contribution (as explained in the preceding section) can be completely different. Nevertheless, we believe that going beyond the standard SW description will not really solve another more essential problem present in this kind of systems. One of the main features of these models is that relaxation occurs by the coarsening of a typical length scale. This length scale reflects the typical size of spatial regions which are ordered into the ground state structure. As relaxation proceeds the configuration of the system approaches that of the ground state. Now, the ground state in this class of systems corresponds to the crystal structure in structural glasses. So the relevant question is: How important is the existence of a crystalline configuration in structural glasses as far as the glass transition phenomenology is concerned? Light scattering measurements point into the direction that relaxation in the under-cooled region does not proceed by crystallization of larger and larger regions and that the structure of the glass is always that of a liquid. This result is in agreement with the very well known fact that some spin glasses with disorder (where there does not exist a crystalline structure) display a behavior similar to structural glasses. Hence we must conclude that the slow dynamics in glasses is not necessarily related to a coarsening process into a single and unique structure. The physical mechanism behind relaxation then must be some kind of entropic search thermally activated but without any kind of growing order in the system. On the other hand the existence of a some kind of coarsening process in the relaxation will preclude the validity of the equiprobability hypothesis, and hence the existence of a flat measure for the free energy. Therefore kinetically constrained models, despite their striking non-equilibrium behavior very similar to that of glasses, cannot be described in a framework as that proposed in the previous section. Nevertheless, a careful study of the constrained Ising chain in terms of the free energy could be certainly interesting to better elucidate this question.

\section{Conclusions}

There is still more work to be done. Most of the lines of research here presented will clearly see a fast development in the next future when our understanding of the validity of general scenarios to describe glassy systems will improve. The most important results we have tried to propose in this paper can be summarized in the form of answers to the following set of selected questions,

- When the $S W$ decomposition is expected to work fairly well? A statistical description of valleys in terms of inherent structures seems to be useful for systems 
where valleys are very narrow (i.e. containing a exponentially large number of configurations scaling like $\exp (\alpha N)$ but with $\alpha \ll 1$ to assure that the entropic contribution to the free energy valley is small) or valleys very large but with very similar shape. The later assumption corresponds to the claim that the distribution of instantaneous frequency modes computed in the harmonic approximation for those valleys are not too broad functions. This is indeed the case for, e.g., BMLJ [29. Obviously, glassy systems where there is a highly heterogeneous distribution of basins of attraction for the inherent structures cannot be described within the usual SW approach.

- How one can improve the standard $S W$ approach? The idea to include the basins of attraction in the dynamical formulation is by supposing that basins are visited according to their free energy. Therefore, in equilibrium at temperature $T$, the probability to visit a valley with free energy $F$ is proportional to the Boltzmann factor $\exp (-\beta F)$ and to their number $g(F, T)=\exp \left(S_{c}(F, t)\right)$ where $S_{c}(F, T)$ defines the configurational entropy. This free-energy measure can be further extended to deal with non-equilibrium processes where the probability to jump among valleys is simply given by the entropic term $g\left(F^{*}, T\right)$ evaluated at the time-dependent threshold free energy $F^{*}=F(t)$. This description offers an interpretation of the violation of $F D T$ in terms of a single timescale given by the effective temperature evaluated at the threshold $1 / T_{\mathrm{e}}(t)=\left(\frac{\partial S_{c}(F, T)}{\partial F}\right)_{F=F^{*}}$. The validity of this flat measure in terms of the free energy in the off-equilibrium regime remains one of the most fascinating problems when trying to have a general theory for slowly relaxing non-equilibrium processes in complex free energy landscapes.

- What is the utility of investigating relatively small systems? As we have stressed in the previous item a description of basins in glassy systems must be done in terms of the free-energy landscape. The appropriate configurational entropy is then a function of both the free energy and temperature. Within the IS formalism an estimate of the free energy of each IS can be done by sampling the IS-space. A good sampling requires that each IS is visited with a finite frequency. If the number of IS is too large this is not possible. Considering that the total number of IS is exponentially large with the volume of the system we conclude that sizes must be modest for the procedure to be implemented. Moreover, in the case of mean-field models, one can do careful checks of the main theoretical assumptions by comparing numerical results with analytical ones. Furthermore, a theoretical analysis of finite $N$ corrections in mean-field systems could give a theoretical framework for activated processes in glassy systems.

- Is the IS formalism relevant for coarsening models? In principle, for systems where a given pattern grows with time the dynamics cannot be expressed in terms of jumps among uncorrelated structures. Therefore, the entropic assumption is not justified and a dynamical measure in terms of the free energy landscape does not hold anymore. In this respect it would be extremely interesting to find a coarsening model where the IS formalism in terms of the free energy works. To our knowledge, such an example has not been yet provided.

In summary, the IS description of dynamics in terms of the energy and, more generally, in terms of the free energy of basins provides the first approximate scheme to deal with the dynamics of complex systems. There are still obscure and not well understood points which hopefully will be progressively clarified in the near future. 
This will provide a more complete comprehension of the main physical mechanisms behind the elusive glass transition problem.

\section{Acknowledgments}

We thank for useful discussions B. Coluzzi, C. Donati, U. Marini Bettolo, E. Marinari, A. Rocco F. Sciortino, M. Sellitto and P. Tartaglia. A special thank goes to F. Sciortino for having given us the data for BMLJ systems. F.R. acknowledges funding from the project PB97-0971 and Acciones Integradas collaboration HI2000-0087.

\section{References}

[1] W. Gotze, in Liquid, Freezing and the Glass Transition, 1989 Les Houches Lectures, Ed. by J. P. Hansen, D. Levesque and J. Zinn-Justin (North-Holland, Amsterdam, 1989).

[2] M. Goldstein, Phys. Rev. 51, 3728 (1969)

[3] F. H. Stillinger and T. A. Weber, Phys. Rev. A25, 978 (1982)

[4] F. H. Stillinger, Science 267, 1935 (1995).

[5] D. J. Wales, M.A. Miller and T.R. Walsh, Nature 394, 758 (1998).

[6] A. Crisanti and F. Ritort, Europhys. Lett. 51, 147 (2000).

[7] A. Crisanti and F. Ritort, Europhys. Lett. 52, 640 (2000).

[8] A. Crisanti and F. Ritort, J. Phys. C (Condensed Matter) 12, 6413 (2000); Physica A 280, 155 (2000).

[9] F. Sciortino and P. Tartaglia, Phys. Rev. Lett. 86, 107, (2001).

[10] A. Crisanti, F. Ritort, A. Rocco and M. Sellitto, Journal of Chemical Physics 113, 10615 (2000).

[11] C. Beck and F. Schlögl, Thermodynamics of chaotic systems:an introduction, Cambridge Nonlinear Science Series 4, Cambridge University Press, 1993.

[12] E. Marinari, G. Parisi and F. Ritort, J. Phys. A (Math. Gen.) 27, 7847 (1994); G. Parisi and M. Potters, J. Phys. A (Math. Gen.) 28, 5267 (1995)

[13] W. Kob and H.C. Andersen, Phys. Rev. Lett. 73, 1376, (1994); Phys. Rev. E 51, 4626 (1995); Phys. Rev. E 52, 4134 (1995). M. Nauroth and W. Kob, ibid. 55, 675 (1997); T. Gleim, W. Kob, and K. Binder, Phys. Rev. Lett. 81, 4404 (1998).

[14] T. R. Kirkpatrick and D. Thirumalai, Phys. Rev. Lett. 58, 2091 (1987); T.R. Kirkpatrick and P.G. Wolynes, Phys. Rev. B36, 8552 (1987); A. Crisanti, H. Horner and H. J. Sommers, Z. Physik B92, 257 (1993).

[15] J. P. Bouchaud, L. F. Cugliandolo, J. Kurchan and M. Mezard in Spin Glasses and Random Fields Ed. by A. P. Young, World Scientific, Singapore, 1998.

[16] M. Mezard and G. Parisi, Phys. Rev. Lett. 82, 747 (1998); J. Chem. Phys. 111, 1076 (1999).

[17] M. Mezard, Physica A, 265, 352 (1999); B. Coluzzi, M. Mezard, G. Parisi and P. Verrochio, J. Chem. Phys. 111, 9039 (1999).

[18] I. Ohmine, H. Tanaka and P.G. Wolynes, J. Chem Phys. 89, 5852 (1998); H. Tanaka, Nature 380, 328 (1996); S. Sastry, P.G. Debenedetti and F. H. Stillinger, Nature 393, 554 (1998);

[19] W. Kob, F. Sciortino and P. Tartaglia, Europhys. Lett. 49, 590 (2000).

[20] This expression, and its differential counterpart, is valid in the equilibrium regime. In the nonequilibrium regime its validity is based the existence of an equiprobability principle which asserts that all basins with the same free energy are equiprobable 28. Se also S. Franz and M. Virasoro, J. Phys. A (Math. Gen.) 33, 891 (2000).

[21] A. Crisanti, E. Marinari, F. Ritort and A. Rocco cond-mat/0105391, (2001).

[22] S. F. Edwards and A. Mehta, J. Phys. (France) 50, 2489 (1989).

[23] R. Monasson and O. Pouliquen, Physica A 236, 395 (1997).

[24] M. Sellitto, in this volume.

[25] A. Barrat, J. Kurchan, V. Loreto, M. Sellitto, Phys. Rev. Lett. 85, 5034 (2000); Phys. Rev. E 63, 051301 (2001)

[26] D. S. Dean and A. Lefevre, Phys. Rev. Lett. 86, 8639 (2001); A. Lefevre and D. S. Dean, Eur. Phys. J. B 21, 121 (2001)

[27] See for example Spin Glass Theory and Beyond, by M. Mézard, G. Parisi and M. Virasoro (World Scientific, Singapore 1987); Spin Glasses Fischer, K.H, Hertz, J.A. (Cambridge University Press, 1991).

[28] A. Crisanti and F. Ritort cond-mat/0102104, (2001). 
[29] F. Sciortino, private communication (2001). 